\title{
Verhalten und Resistenz mariner Diatomeen gegenüber Veränderungen der Salzkonzentration
}

\author{
HeRmanN FISCHER \\ Botanisches Institut der Universität Bonn
}

\begin{abstract}
Responses and resistance of marine diatoms to changes in salinity. Diatoms of littoral regions are often exposed to considerable salinity fluctuations. This fact suggests that these unicellular organisms exhibit osmotic resistances similar to those found in larger multicellular algae. The responses of typical representatives of the littoral diatom flora were studied after transfer into diluted or concentrated sea water. The technique applied made it possible to compare the results with corresponding data from literature obtained on green, brown and red algae of the tidal zone. The total amount of resistance of the diatoms studied may reach the values obtained on these multicellular algae or may be lower. The results imply the presence of mechanisms capable of flexible osmotic adjustments to salinity changes. In addition to their osmotic resistance sensu strictu, protection from the ill effects of extreme salinities may be of importance, for example, through the formation of jelly, variations in population density and perhaps the ability to migrate into more suitable conditions. All species tested have shown an ability for osmoregulation or for passively tolerating changes in internal osmoconcentration. Some may tolerate plasmolysis even for prolonged periods. Diatoms exhibiting quantitative and qualitative differences in osmotic resistance may occur in the same microhabitat.
\end{abstract}

\section{EINLEITUNG}

Zu den abiotischen Faktoren, deren biologische Wirkungen das Hauptthema unserer Tagung sind, gehört der Salzfaktor, über dessen allgemeine Bedeutung für die Organismen ich in diesem Kreise nicht zu sprechen brauche. Ich möchte vielmehr über Versuche zur Wirkung veränderter Außenkonzentrationen auf Diatomeen berichten, und zwar speziell auf solche des Litorals. Diatomeen sind quantitativ bedeutende Komponenten der Biocoenosen des Gezeitenbereichs, sie fallen infolge ihrer Massenentwicklung auf den Wattoberflächen oder als Epiphyten auf größeren Algen schon durch ihre braune oder olivgrüne Farbe auf. Schon vor längerer Zeit wurde bei einem Aufenthalt an der Station auf dem Ellenbogen bei List meine Aufmerksamkeit auf die Frage gelenkt, wie die Diatomeen reagieren, wenn die Salzkonzentration an ihrem Standort bei Ebbe, insbesondere durch starke Evaporation bei warmem Wetter, steigt oder wenn sie durch Regen oder einströmendes Süßwasser rasch herabgesetzt wird. Diese Frage ist nicht nur ökologisch interessant, sondern auch wegen einer zellphysiologischen Eigentümlichkeit der Diatomeen, von der am Schluß die Rede sein soll. 


\section{METHODIK}

Die Untersuchungsmethode war stets die gleiche: Das Versuchsmaterial wurde in verschlossene Schälchen mit abgestuften Meerwasserkonzentrationen gebracht; sofort, und in bestimmten Zeitabständen danach, wurden die Wirkungen der erhöhten und herabgesetzten Salzkonzentrationen festgestellt. Meist wurden Stufen im Abstand von 0,1 oder 0,2 der normalen Meerwasserkonzentration verwendet (im folgenden als

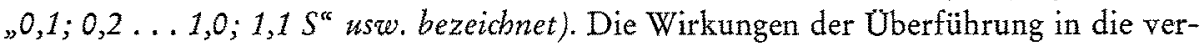
änderte Konzentration wurden festgestellt, indem die Anzahl der jeweils intakten, plasmolysierten und abgestorbenen Zellen von $25 \mathrm{zu} 25 \%$ geschätzt, in einigen Fällen auch ausgezählt wurde.

\section{UNTERSUCHUNGSERGEBNISSE}

Bringt man eine Pflanzenzelle in eine hypertonische Lösung, so tritt Plasmolyse ein, welche wieder zurückgeht, wenn die Konzentration des Zellsaftes, etwa durch Stoffaufnahme von außen, erhöht wird. Beide Vorgänge, Plasmolyse wie Deplasmolyse, können in der Gezeitenzone unter natürlichen Bedingungen ablaufen. Die Resistenz der Zellen gegenüber Erhöhung der Salzkonzentration wird entscheidend davon bestimmt sein, ob eine Plasmolyse eintritt oder nicht, und davon, wie im ersten Fall die Plasmolyse ertragen wird.

Um die Resistenz zuverlässig feststellen zu können, wurden solche Diatomeen ausgewählt, die in sehr großer Individuenzahl vorkommen; es handelt sich dabei um Formen, die möglichst große Verschiedenheiten im Bau und hinsichtlich ihres Standortes aufweisen. Zum Vergleich der Reaktionen meiner Versuchsobjekte habe ich in einem Diagramm osmotische Resistenz und Plasmolyserückgang für alle diejenigen Diatomeen zusammengefaßt, die ich gründlicher untersucht habe. Die Versuchsergebnisse stammen aus verschiedenen Jahren und beziehen sich auf Material von List, Roscoff und Helgoland. Die Arbeitsbedingungen waren an allen drei Orten insofern ähnlich, als die Arbeitsräume kühl und schattig waren, so daß ihre Temperatur etwa bei $20^{\circ} \mathrm{C}$ lag. Freilebende Diatomeen wurden in kleinen Schalen gehalten, Epiphyten zusammen mit den Algen, auf denen sie wuchsen, und zwar in fließendem Seewasser.

Ein Mangel in der Darstellung der Abbildung 1 liegt darin, daß sie die Verhältnisse für alle Arten zwar nach längerem Aufenthalt in der Versuchslösung wiedergibt, aber nicht nach der gleichen Zeit, weil die Versuche nicht von vornherein auf einen solchen Vergleich ausgerichtet waren. Die Blöcke des Diagramms geben den Zustand für die verschiedenen Diatomeen nach 14 bis 24 Stunden wieder; d. h. es könnte bei einigen der Prozentsatz der abgestorbenen oder der deplasmolysierten Zellen noch höher gewesen sein. Diese Unterschiede sind bei längeren Versuchszeiten weniger bedeutend, als es mir anfangs schien; besonders der Plasmolyserückgang erfolgt in den ersten Stunden nach der Übertragung am raschesten, und auch die Schädlichkeitsgrenze ist (besonders bei Seewasserverdünnungen) oft recht scharf, so daß nach 2 bis 4 Stunden der Zustand erreicht wird, der sich noch am anderen Tage findet.

Die Blöcke 1 bis 4 zeigen das Verhalten von Formen, die als Aufwuchs auf Algen 
weit verbreitet sind; besonders Licmophora (2) und Grammatophora (3) bilden dichte Rasen, aber auch Striatella (1) und Achnanthes (4) treten in so großer Menge auf, wie es für die Untersuchung wünschenswert ist. Man sieht, daß für Striatella, Licmophora und Grammatopbora eine Verdünnung des Seewassers auf etwa die Hälfte kritisch ist,

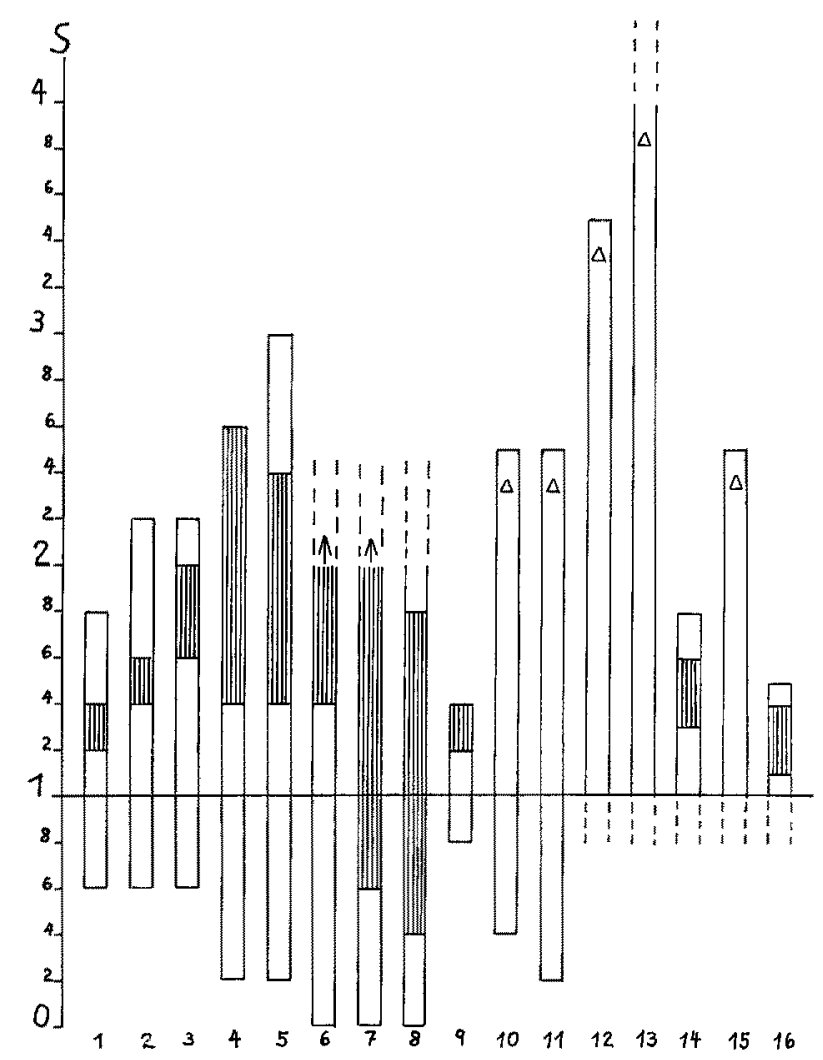

Untersucbte Diatomeen (in Klammern: Zeit nach der Übertragung in die Versucbslösungen):

1 Striatella unipunctata (LYNGB.) AG. (15 b)

2 Licmopbora paradoxa (LYNGB.) Ag. (15 b)

3 Grammatophora marina (LYNGB.) KÜTZ. $(15 \mathrm{~b})$

4 Achnanthes longipes AG. (24 b)

5 Melosira dubia KÜTZ. (24b)

6 Melosira moniliformis (Mült.) $A_{G}$, nach $24 b$ in $1,0 \mathrm{~S}(14 \mathrm{~b})$

7 desgl., aus dem Standortwasser $(14 \mathrm{~b})$

8 desgl., nach 24 h in Süßwasser $(14 h)$

9 Bacillaria paradoxa GMEL. (19 b)

10 Gyrosigma balticum (EHR.) Cl. (21 b)

11 Amphora ct. arenicola GRUN. (21 b)

12 Scolioplemra tumida (BRÉB.) RABH. (24 b)

13 desgl., $24 b$ in $2,0 \mathrm{~S}, 24 b$ in $3,0 \mathrm{~S}$ (danach 24 b)

14 Nitzschia closteritum (EHR.) W. SM. $(18$ b)

15 Tropidoneis lepidoptera GREG. $(24$ b)

16 Donkinia recta var. intermedia Perag. $(51 / 2 b)$

Abb. 1: Osmotische Resistenz von Diatomeen. Die Blöcke repräsentieren Konzentrationen (als n-fach Meerwasser ausgedrüdkt), in denen nach der bei den einzelnen Arten unten angegebenen Zeit noch mindestens $50 \%$ der Zellen am Leben waren. Offene Blöcke und unterbrochene Linien: Resistenzgrenze wurde im Versuch nicht erreicht, weil höhere bzw. niedrigere Konzentrationen nicht untersucht wurden. Die mit senkrechten Strichen ausgefullten Teile der Blöcke bezeichnen den Konzentrationsbereich, in dem zu Beginn und am Ende der Versuchszeit die Grenzplasmolyse lag, ihre Länge läßt also den Rückgang der Plasmolyse erkennen. Zeichenerklärung: $\uparrow$ Deplasmolyse war eingetreten, jedoch wurde bei höheren Konzentrationen nicht mehr geprüft es ist daher möglich, daß die Plasmolyse noch weiter zurückging. $\triangle$ Grenzplasmolyse wurde nicht gemessen. Die Plasmolyse ging hier rasch bis in den Bereich der schädlichen Konzentrationen zurïck

sie können aber eine plötzliche Erhöhung auf fast das Doppelte oder noch mehr vertragen. Eine gewisse Erhöhung des osmotischen Wertes findet statt, die aber nicht bis an die Resistenzgrenze geht; im oberen Konzentrationsbereich überleben die Zellen im plasmolysierten Zustand. Sie sind sich in ihrem Verhalten untereinander ähnlich, und 
man könnte zunächst vermuten, hier den physiologischen Typ der Aufwuchsdiatomeen gefunden zu haben.

Es war mir aber schon bei meinen Beobachtungen in List aufgefallen, daß bei Acbnanthes longipes, die man oft in den Rasen von Licmophora und Grammatophora findet, die Plasmolyse weiter zurückging und offenbar auch die Resistenz größer war. Das gleiche sah ich in Roscoff, hatte aber erst auf Helgoland genügend Material zur Verfügung für eine quantitative Beurteilung. Man ersieht aus Block 4, daß Achnanthes nicht nur gegen Konzentrationssteigerung, sondern auch gegen Konzentrationsherabsetzung viel widerstandsfähiger ist als die drei anderen Arten vom gleichen Standort. Die sehr viel größere Fähigkeit zur Regulation des osmotischen Wertes ist deutlich.

Die Achnanthes-Proben, die sich nicht anders verhielten, als es früher an Material von natürlichen Standorten festgestellt wurde, stammten aus Zuchtbecken des Aquariums der Biologischen Anstalt Helgoland. Hier fand sich auch Melosira dubia (5) in größerer Menge. Auch sie kann im osmotischen Wert der erhöhten Außenkonzentration beträchtlich folgen, ihre Hypotonieresistenz ist so groß wie die von Achnanthes, die Hypertonieresistenz noch größer. Mit ihr zusammen lebte im Algendetritus die merkwürdige Bacillaria paradoxa, welche durch die raschen Gleitbewegungen ihrer Kolonien einzigartig ist. Ein Blick auf Block 9 zeigt für Bacillaria eine viel geringere Resistenz und nur schwachen Konzentrationsausgleich in der Zelle. Vergleicht man sie mit Melosira dubia, so finden sich wieder (mindestens an diesem künstlichen Standort) zwei sehr ungleiche Gefährten vereinigt.

Melosira moniliformis (Abb. 1; 6, 7, 8) ist eine großzellige Aufwuchsform, die auch im Brackwasser massenhaft auftritt. Ich fand sie fast in Reinkultur am Überlauf aus einem brackigen, kaum salzig schmeckenden Teich in einem Park an der bretonischen Küste. Block 7 zeigt das Verhalten im Standortwasser: auch eine Überführung in reines Süßwasser (Leitungswasser) schadet ihr nicht, Konzentrationserhöhungen weichen die Zellen weitgehend aus, und zwar tun sie dies sehr rasch. Genaue Messungen habe ich nur bis 2,0 S gemacht; der Pfeil deutet an, daß die Plasmolyse auch noch bei höheren Konzentrationen zurüdkgehen dürfte; die Resistenzgrenze liegt nach einigen Beobachtungen bei 3,0 S.

Hält man die Alge 24 Stunden in normalem Seewasser (6), so erreicht sie einen osmotischen Wert, der über der neuen Außenkonzentration liegt, bei Uberführung solcher Zellen in höhere Konzentrationsstufen tritt, wie zu erwarten, eine weitere Anpassung an die Außenkonzentration ein. Trotzdem bleibt die Hypotonieresistenz die gleiche. Auch hier führt eine Überführung in Leitungswasser zu keinerlei feststellbaren Schäden.

Durch diese Eigenschaft wird der in Block 8 dargestellte Versuch möglich, bei welchem die Zellen 24 Stunden in Leitungswasser gehalten worden waren. Dabei wird die plasmolytische Grenzkonzentration auf $0,4 \mathrm{~S}$ gesenkt, sie liegt damit ebenso hoch über der Außenkonzentration wie nach Aufenthalt in normalem Seewasser. Hier wurde zum erstenmal bei Melosira moniliformis eine Begrenzung der Deplasmolyse innerhalb der Versuchszeit von 14 Stunden sichtbar. Die Zellen bleiben aber auch jetzt noch bei doppelter Seewasserkonzentration intakt.

Aus den Versuchen mit verschiedener Anfangskonzentration des Milieus folgt, daß diese Brackwasserdiatomee offenbar sehr gut an Konzentrationswechsel angepaßt ist; 
sie reagiert wie $A$ chnanthes mit Anderung der Innenkonzentration und vermeidet dadurch weitgehend den Plasmolyseeintritt. Man darf wohl annehmen, daß sich auch bei Achnantbes longipes und bei Melosira dubia durch Vorbehandlung mit verdünntem Seewasser die Zellsaftkonzentration herabsetzen ließe.

Die in der rechten Hälfte der Abbildung 1 zusammengefaßten Diatomeen sind bewegliche Formen der Wattoberfläche; sie bilden keine Kolonien. Gyrosigma balticum (10) und eine Ampbora (11) kamen zusammen in großer Zahl auf dem Schlick eines Watts unweit Roscoff vor. Sie gleichen sich auch im Versuch: Bei hypertonischen Konzentrationen zeigen die Zellen zunächst Plasmolyse, die aber bald zurückgeht. Übrigens läßt sich die Plasmolyse nicht bei allen Zellen auf den ersten Blick erkennen; man kann daher nicht mit Sicherheit die wirkliche Zahl der plasmolysierten Zellen rasch schätzen. Ich habe hier und bei anderen Zellen daher nicht den plasmolytischen Grenzwert festgestellt. Charakteristisch ist, daß die Plasmolyse bis fast zu der Konzentration hinauf zurückgeht, die für die Zellen schon schädlich sind. Offenbar liegt hier wie bei Achnanthes longipes und bei Melosira moniliformis die Anpassung an das wechselnde Milieu im Konzentrationsausgleich, nicht in einer Resistenz gegen Plasmolyse. Die so erreichte Hypertonieresistenz liegt höher als bei den Aufwuchsformen mit geringem Konzentrationsausgleich; auch die Hypotonieresistenz ist beträchtlich.

Eine Charakterart des Schlickwatts der Nordsee ist Scoliopleura tumida (12). Sie gehört zu dem gleichen physiologischen Typ mit starkem Plasmolyserückgang und erreicht unter allen von mir untersuchten Arten die höchste Resistenz. Die Zellen überleben zwei Wochen im deplasmolysierten Zustand und sind offenbar völlig gesund in doppelt und dreifach konzentriertem Seewasser. Erst bei noch höheren Konzentrationen wird die kritische Grenze überschritten, und diese Grenze ist wieder ziemlich scharf, indem nach Überführung in 3,5 S schon in der ersten Stunde die Zellen abzusterben beginnen. Jedoch läßt sich auch Scoliopleura an höhere Konzentrationen gewöhnen: Aus normalem Seewasser für 24 Stunden in doppelt konzentriertes und daraus für weitere 24 Stunden in dreifach konzentriertes gebracht, zeigte sie erst bei $4,0 \mathrm{~S}$ vorübergehend Plasmolyse, die schon nach 15 Minuten zurüdkging. Selbst am nächsten Tage waren die meisten Zellen in 4,0 S noch voll vital (13). Auch hier war also die Zellsaftkonzentration - und parallel dazu-die Hypertonieresistenz erheblich gestiegen.

Leider habe ich bei meinen ersten Beobachtungen in List, die für mich eine erste Orientierung auf diesem Gebiet waren, keine Versuche mit herabgesetzter Konzentration gemacht. So kann ich nicht beweisen, daß analog zu anderen Arten bei Scoliopleura der hohen Hypertonieresistenz entsprechend auch eine große Widerstandsfähigkeit gegen Aussüßung vorhanden ist. Ich habe damals mein Augenmerk vor allem darauf getichtet, ob die verschiedenen Formen des gleichen Standorts auf Konzentrationserhöhung in der gleichen Weise reagieren. Daß dies nicht der Fall zu sein braucht, zeigen die Blöcke 14, 15 und 16.

Nitzschia closterium (14) ist eine kleine Diatomee, die häufig und in großer Individuenzahl auf Schlick und Sand zu finden ist. Ihre Resistenzbreite und osmotische Regulation bei Konzentrationserhöhung sind nicht größer als bei Striatella. Schließlich zeigen Blodk 15 und 16 zwei häufige Diatomeen des Sandwatts aus der gleichen Probe: Tropidoneis lepidoptera deplasmolysiert leicht und ist osmotisch recht resistent, während Donkinia recta var. intermedia nur sehr beschränkte Fähigkeit zum Konzentra- 
tionsausgleich zeigt und schon eine Heraufsetzung der Seewasserkonzentration um die Hälfte nicht mehr verträgt. Ihre Resistenz ist eher noch geringer, als aus dem Vergleich hervorgeht, weil Block 16 den Zustand nach $51 / 2$ Stunden darstellt, nicht wie sonst den vom folgenden Tage. Es ist anzunehmen, daß schon jede Ebbe an warmen Sommertagen für Donkinia kritisch werden könnte.

Über die Salzgehaltsschwankungen, denen die Diatomeen im Gezeitenbereich tatsächlich ausgesetzt sind, kann ich leider nichts aussagen; wohl aber läßt sich ihre Resistenzbreite mit der von Grün-, Braun- und Rotalgen vergleichen. Durch Brebls (1958) Untersuchungen von verschiedenen Küsten haben wir ein gutes Bild über die osmotische Resistenz einer großen Anzahl von Algen. In der Regel ertragen Algen der Gezeitenzone nach plötzlichem Milieuwechsel Konzentrationen zwischen 0,1 und 3,0 S 24 Stunden lang, zum Teil sogar von reinem Süßwasser bis 4,0 S. In diesem weiten Bereich bleibt, falls auch ihre Hypotonieresistenz entsprechend ist, von den Diatomeen der Wattoberfläche Scoliopleura tumida am Leben, andere (Gyrosigma balticum und Amphora cf. arenicola) nur zwischen $0,2(0,4)$ und etwa 2,5 S. Diese Diatomeen deplasmolysieren so rasch, daß es in der Natur wahrscheinlich gar nicht zur Plasmolyse kommt. Thre hohe Resistenz gegen Konzentrationssteigerungen beruht offenbar darauf, daß die Zelle der Steigerung folgt, Hypertonie also weitgehend vermieden wird. Wir haben daher keine echte Hypertonieresistenz vor uns, sondern eine Resistenz der Zelle gegen hohe Innenkonzentration. Auch unter den epiphytischen Diatomeen sind diejenigen am resistentesten, die ihren osmotischen Wert am besten regulieren können (Achnanthes longipes und Melosira moniliformis; bei Melosira dubia ist eine nennenswerte Osmoregulation mit einer deutlichen Plasmolyseresistenz verbunden).

Auch die vielzelligen Algen der Gezeitenzone sind mehr oder weniger in der Lage zu deplasmolysiern, also faktisch die Plasmolyse zu verhindern. Andere vermeiden den Plasmolyseeintritt dadurch, daß sie von vornherein eine Zellsaftkonzentration haben, die etwa doppelt so hoch ist wie die des Seewassers; schließlich gibt es auch Algen mit erheblicher Plasmolyseresistenz. Plasmolyse am Standort ist bei Grünalgen und sogar bei Rotalgen, die sonst sehr hypertonieempfindlich sind, beobachtet worden. Sie kommt auch bei manchen Diatomeen vor (BrockMANN 1950 und eigene Beobachtungen). Unter extremen Bedingungen lebende Algen kombinieren gute Osmoregulation und hohe Plasmolyseresistenz: Enteromorpha clathrata aus Salzpfannen in Kalifornien, wo die Bedingungen noch wesentlich härter sind als im Gezeitenbereich, bringt es dabei auf eine Resistenz von 0,0 bis 5,0 S (Biebl 1956). Man darf für Diatomeen solcher Standorte ähnliches erwarten.

Algen des Sublitorals vertragen wesentlich geringere Schwankungen als die des Litorals, ihnen fehlt auch weitgehend die Fähigkeit zur Deplasmolyse. Ihre Resistenzgrenzen liegen $\mathrm{zw}$ ischen 0,4 und 1,8 S, zum Teil nur zwischen 0,6 und 1,3 $\mathrm{S}$. In diesem Bereich liegt von unseren Epiphyten Striatella $(0,6$ bis $1,8 \mathrm{~S})$, während Licmophora und Grammatophora $(0,6$ bis 2,2$)$ in ihrer oberen Resistenzgrenze den Algen der Niedrigwasserlinie entsprechen $(0,3$ bis 2,2 S). Diese Diatomeen werden mit den Algenthalli, auf denen sie wachsen, bei Ebbe vielfach freigelegt, und auch so ausgesprochene Litoralformen wie Nitzschia closterium und Donkinia recta var. intermedia ertragen nur 1,8 bzw. 1,5 S, obwohl sie regelmäßig der Gefahr der Versalzung (und der Aussüßung) ausgesetzt zu sein scheinen. 


\section{DISKUSSION}

Wenn wir auch nicht wissen, welchen osmotischen Belastungen die Algen am Standort wirklich ausgesetzt sind, so dürfen wir doch fragen, wie manche Diatomeen mit einer vergleichsweise geringen Resistenz auskommen können. Sieht man davon $a b$, daß die Versuche im allgemeinen sprunghafte Konzentrationsänderungen brachten, die in der Natur nicht die Regel sind, und daß die Zeit, während derer die Diatomeen tatsächlich frei liegen, of nur kurz ist, so haben die einzelligen Organismen den Vorteil der raschen Vermehrung, der sich auswirken kann, wenn wenigstens ein Teil der Population überlebt. Für die epiphytischen Diatomeen ist es ferner von Vorteil, daß sie Wasser zwischen sich festhalten. Sie sind daher vor Konzentrationsänderungen an ibrer Zelloberfläche mehr oder weniger geschützt, sobald der Bewuchs eine gewisse Dichte erreicht hat, wie es gerade bei Licmophora und Grammatophora in der Hauptvegetationszeit die Regel ist. In ähnlicher Weise wirken die Gallerthüllen, die von vielen Diatomeen, auch von einzeln lebenden, ausgeschieden werden. Auf der Wattoberfläche bilden sie zusammenhängende gallertige Häute, die allerdings unter extremen Verhältnissen auch papiertrocken werden können.

Vielleicht kommt den Wattdiatomeen auch ihre Beweglichkeit zugute, die nach Nultsch (1962) etwa doppelt so groß ist wie bei den am schnellsten kriechenden Süßwasserarten. Wattdiatomeen führen Vertikalbewegungen aus, deren Ursachen noch Gegenstand der Diskussion sind (Peruss 1960). Sie kommen yielfach bei Ebbe an die Oberfläche, wo sie assimilieren können, ohne fortgespült $\mathrm{zu}$ werden. Daneben gibt es aber auch Beobachtungen, nach denen sich Diatomeen gerade von der trodknen Wattoberfläche zurïckziehen (BrockMANN 1950, p. 9; Gessner 1955, p. 248). Unsere Kenntnisse hierüber sind noch sehr unvollständig, und ich möchte nur auf die Möglichkeit hinweisen, daß die Beweglichkeit bei einigen Diatomeen zur Flucht unter die Oberfläche dienen könnte, etwa bei Nitzschia closterium und Donkinia recta var. intermedia.

Sehr auffallend ist in diesem Zusammenhang, daß die außerordentlich bewegliche Bacillaria paradoxa in meinen Versuchen die geringste Resistenz hatte. Sie gehört zu den wenigen Diatomeen, die im Meer und im Süßwasser vorkommen. KarSTEN (1899), von dem die ersten Versuche über die Wirkung der Außenkonzentration auf Meeresdiatomeen stammen, hat an Bacillaria paradoxa aus der Kieler Bucht eine etwas höhere Resistenzspanne gefunden. Er arbeitete mit allmählichen Konzentrationssteigerungen und -senkungen. Die Zellsaftkonzentration lag entsprechend dem geringeren Salzgehalt der Ostsee niedriger. Unterschiedliche osmotische Werte und Verschiebung der Resistenzgrenzen finden sich ïberall, wenn man die gleichen Algenarten aus Meeresgebieten mit verschiedenem Salzgehalt vergleicht (SCHWENKE 1958).

Uber die physiologischen Grundlagen der Resistenz sind unsere Vorstellungen noch recht liickenhaft. Die Plasmolyse wird keine irreversible Schädigung bedeuten, wenn sich das Cytoplasma von der Wand glatt ablöst. Durch die Schrumpfung wird das Gefüge des Protoplasten und sein Stoffwechsel gestört werden können, besonders wenn diese länger andauert. HorFmanN (1959) hat speziell die Bedeutung der Regulation des Turgors und damit des Normalzustandes der Pflanzenzelle betont. Andererseits führt Aussïßung bei mangelnder Regulation nicht nur zum Überschwemmen des Plasmas mit Wasser, sondern auch zum Anschwellen der Vakuole und im Extremfall 
zum Platzen der Zellwand. Sehr zarte Zellwände können daher die Ursache hoher Hypotonieempfindlichkeit sein, wie es bei der mediterranen Diatomee Biddulphia titiana der Fall ist, die zwar eine gewisse Resistenz gegen erhöhte Konzentrationen hat, aber die Turgordehnung so wenig verträgt, daß sie schon in 0,95 $\mathrm{S}$ abzusterben beginnt (Höfler 1963). Solche Organismen wird es im Gezeitenbereich nicht geben. Alle Gezeitendiatomeen, auch die am wenigsten widerstandsfähigen, haben eine vergleichsweise hohe Resistenzspanne. Im allgemeinen liegen die Unterschiede in der Resistenz wohl weniger im mikroskopisch erkennbaren Bau der Zellen begründet schon nahe verwandte Formen können sich sehr ungleich verhalten -, als in Eigenschaften des Protoplasmas.

Der wirksamste Faktor ist dabei, wie wir sahen, die osmotische Regulation. Vor zwei oder drei Jahrzehnten hätten wir den Plasmolyserückgang mariner Algenzellen ohne Zögern als eine passive Salzendosmose verstanden, durch welche der auf nicht permeierenden gelösten Stoffen beruhende osmotische "Grundwert" entsprechend der Außenkonzentration und den Erfordernissen der Turgorregulation verändert wird. Wir wissen aber heute, $\mathrm{daB}$ auch für Algenzellen diese Vorstellung nicht ausreidt, und wir haben durch Kesseler $(1959,1962)$ gerade auch für die Gezeitenalge Chaetomorpha linum Beweise für die spezifische Rolle einzelner Ionen bei der Turgorregulation erhalten. Es ist noch offen, welcher Anteil bei den Diatomeen aktiven Regulationsvorgängen einerseits und passivem. Stoffaustausch andererseits zukommt. Diatomeen sind dadurch noch besonders interessant, daß viele unter ihnen im Gegensatz zu anderen Pflanzenzellen in hypertonischen Zuckerlösungen rasch deplasmolysieren, also nach herkömmlicher Auffassung eine hohe Zuckerpermeabilität haben, zu der eine entsprechende Salzpermeabilität parallel laufen könnte. Meine Untersuchungen, die Stichproben zum Problem der osmotischen Resistenz darstellen, können jedoch über den Mechanismus der osmotischen Regulation bei Diatomeen nichts aussagen.

\section{ZUSAMMENFASSUNG}

1. Vergleichende Untersuchungen an Diatomeen von verschiedenen Standorten der Gezeitenzone ergaben in allen Fällen eine beträchtliche osmotische Resistenz. Diese kann für die einzelnen Arten sehr unterschiedlich sein. Die festgestellten Resistenzspannen erreichen zum Teil diejenigen, die aus der Literatur für vielzellige Algen des Litorals bekannt sind, zum Teil liegt die Resistenz aber in engeren Grenzen.

2. Unter den Eigenschaften, mit denen sich die Diatomeen auch gegenüber starken Konzentrationsschwankungen behaupten können, tritt ihre Fähigkeit zur Erhöhung und Verminderung der Zellsaftkonzentration besonders hervor. Sie ist spezifisch verschieden stark ausgeprägt und wird durch Plasmolyse- und Turgorresistenz ergänzt. Möglicherweise werden die Zellen ferner durch ihre große Populationsdichte, Gallertausscheidungen und aktive Bewegungen vor der kritischen osmotischen Beanspruchung geschützt.

3. Für die Diatomeen des gleichen Standorts ist nicht ein bestimmter Resistenztyp charakteristisch; es können vielmehr Formen mit verschiedenem Verhalten am gleichen Ort vorkommen. 


\section{ZITIERTE LITERATUR}

BIEBL, R, 1956. Zellphysiologisch-ökologische Untersuchungen an Enteromorpha clatbrata (Roth) Greville. Ber. disch. bot. Ges. 69, 75-86.

- 1958. Temperatur- und osmotische Resistenz von Meeresalgen der bretonischen Küste. Protoplasma 50, 217-242.

Brockmann, C., 1950. Dic Watt-Diatomeen der schleswig-holsteinischen Westküste. Abh. senckenb. naturf. Ges. 478, 26 pp.

Frscher, H., 1952. Uber das Verhalten einiger Watt-Diatomeen in hypertonischen Lösungen. Ber. dtsch. bot. Ges. 65, 218-228.

- 1963. Zur osmotischen Resistenz von Diatomeen der Gezeitenzone. Protoplasma 57, 344-353.

Gessner, F., 1955. Hydrobotanik I. Deutscher Verlag der Wissenschaften, Berlin, 517 pp.

HörLer, K., 1963. Zellstudien an Biddulphia titiana Grunow. Protoplasma 56, 1-53.

Hoffmann, C., 1959, Études écologiques et physiologiques de quelques algues de la mer baltique. Écologie des Algues marines (Coll. internat. CNRS, Dinard 20.-28. Sept. 1957) Paris, pp. 205-218.

Karsten, G., 1899. Die Diatomeen der Kieler Bucht. Wiss. Meeresuntersuch., Abt. Kiel, N. F. 4, 17-205.

KESSELER, H., 1959. Mikrokryoskopische Untersuchungen zur Turgorregulation von Chaetomorpha linum. Kieler Meeresforsch. 15, 51-73.

- 1962. Beziehungen zwischen Atmung und Turgorregulation von Chaetomorpha linum. Helgol. Wiss. Meeresunters. 8, 243-256.

Nultsch, W., 1962. Uber das Bewegungsverhalten der Diatomeen. Planta 58, 22-33.

Perkins, E. J., 1960. The diurnal rhythm of the littoral diatoms of the river Eden estuary, Fife. J. Ecol. 48, 725-728.

SCHWENKE, H., 1958. Uber die Salzgehaltsresistenz einiger Rotalgen der Kieler Bucht. Kieler Meeresforsch. 14, 11-22.

\section{Diskussion im Anschluß an den Vortrag FISCHER}

Levring: Ist etwas darüber bekannt, wie sich Atmung und Photosynthese mit geänderten osmotischen Bedingungen ändern?

FISCHER: Gaswechselmessungen sind meines Wissens im Zusammenhang mit der Osmoregulation weder an marinen noch an limnischen Diatomeen gemacht worden.

SCHLIEPER: Soweit ich verstanden habe, wurde nur die genetische artspezifische Resistenz mariner Diatomeen direkt gemessen. Die individuelle Adaptationsfähigkeit wurde dagegen nicht untersucht. Ist das richtig?

Fischer: Die Untersuchungen beziehen sich nur auf „Anpassung“ innerhalb der kurzen Versuchszeit; sie lassen keine Schlïsse darüber $\mathrm{zu}$, wie weit sich die Diatomeen individuell an veränderte Salzkonzentrationen anpassen können. Man wird wohl bei Formen mit hoher Resistenz und guter Osmoregulation eine entsprechende Adaptation vermuten dürfen.

Overbeck: Gibt es physiologisch verschiedene Rassen von Diatomeen? Bacillaria paradoxa und Nitzscbia closterium kommen im Hiddenseer Brackwasser in stark ausgesüßtem Bradkwasser vor.

Frscher: Bei Bacillaria paradoxa könnte es verschiedene Formen geben, die vielleicht genetisch fixiert sind und im Brackwasser beziehungsweise im Süßwasser leben. In den Untersuchungen von Karsten (1899) hatte Bacillaria paradoxa aus der Kieler Bucht niedrigere osmotische Werte als mein Helgoländer Material. 